\title{
Evaluation of the Agreements of the National Reforestation Program in the Santiago de Quito, Palmira, Pistishi and Compud parishes, Chimborazo Province
}

Memories II International

Congress Forests and

Agroforestry for the 21st

Century

Corresponding Author:

A. Huisha

misterisaias123@hotmail.com

Published: 21 January 2021

Production and Hosting by

Knowledge E

(c) A. Huisha et al. This article is distributed under the terms of the Creative Commons Attribution License, which permits unrestricted use and redistribution provided that the original author and source are credited.
S OPEN ACCESS

\section{Evaluación de los Convenios del Programa Nacional de Reforestación en las Parroquias Santiago de Quito, Palmira, Pistishi y Compud, Provincia de Chimborazo}

\author{
A. Huisha, V. Espinoza, and G. Aldaz
}

Escuela Superior Politécnica de Chimborazo, Facultad de Recursos Naturales, Carrera de Ingeniería Forestal Riobamba, Ecuador

\section{Abstract}

The objective of this study was to evaluate the Forest Restoration agreements, by means of sampling the equivalent of $10 \%$ of the entire ground surface and $10 \%$ of the total land, in which control points were established in order to estimate the planted area; for the verification of land for reforestation, the control points were issued from the central office, which were located in the field with GPS; then the systematization of the data collected in the field was carried out. In the Palmira parish, the area evaluated showed 39.38 ha (74.94\%) in which there were indications of planting, and 12.84 ha (26.06\%) that did not show signs of planting; 57 farms were evaluated, of which 14 (71.93\%) presented evidence of planting and 16 farms (28.07\%) had no such evidence. In the Pistishi parish, the area evaluated had 36.27 ha (93.81\%) in which there were signs of planting, and 2.39 ha (6.19\%) that did not show signs of planting; 29 farms were evaluated, of which 22 (75.86\%) presented evidence of planting and 2 (24.14\%) had no evidence of planting. The properties evaluated in the Santiago de Quito and Compud parishes were considered for reforestation.

Keywords: forest restoration, inventory, biodiversity, reforestation.

\section{Resumen}

El objetivo del trabajo fue evaluar los convenios de Restauración Forestal, mediante un muestreo equivalente al $10 \%$ de toda la superficie y el $10 \%$ del total de los predios, en los que se establecieron puntos de control con la finalidad de estimar la superficie plantada; para la verificación de predios para la reforestación, desde central fue emitido los puntos de control los mismos que fueron ubicados en campo con GPS; seguidamente se realizó la sistematización de los datos recogidos en campo. En la parroquia Palmira se evalúo un área plantada de 39,38 ha $(74,94 \%)$ en el que se registró indicios de haberse plantado, 12,84 ha (26,06\%) no presento indicios de plantación; se evaluaron 57 predios de los cuales 14 (71,93\%) presentó plantaciones y 16 predios (28,07\%) no se registra indicios de plantación. En ella Parroquia Pistishi se evalúo un área de 36,27 ha $(93,81 \%)$ en el que se registró indicios de plantación, 2,39 ha $(6,19 \%)$ no presento indicios de plantación; se evaluaron 29 predios de los cuales 22 (75,86\%) presentó evidencias de plantación y 2 (24,14\%) no se registra indicios de plantación. Los predios evaluados en las parroquias Santiago de Quito y Compud fueron consideraron para la reforestación. 
Palabras Clave: restauración forestal, inventario, biodiversidad, reforestación.

\section{Introducción}

Desde años atrás se están realizado esfuerzos para recuperar ecosistemas boscosos tanto en Europa como en América la motivación de recuperar los ecosistemas boscosos se ha ampliado y ahora incluye la recuperación de la biodiversidad asociada a los bosques y los servicios que proveen estos ecosistemas a la humanidad [1]. La restauración forestal requiere integración de aspectos biológicos y ecológicos, pero también sociales, económicos, políticos, administrativos y legales; es decir, es una disciplina que abarca otras disciplinas que, de hecho, mira más allá del ecosistema degradado. En Ecuador la Restauración forestal se define como estrategia de manejo que tiene como objetivo restaurar el bosque a su estado natural tanto en sus funciones como estructura y composición [2].

El estado ecuatoriano, para el cumplimiento del Plan Nacional de Reforestación en coordinación con el Ministerio del Ambiente suscribió varios convenios de Restauración Activa con 46 Gobiernos Autónomos Descentralizados (GAD) de todo el país, con el propósito de recuperar la cobertura vegetal, a través de un manejo integral del paisaje forestal, lo que permitirá a más de conservar los recursos naturales, mejorar las condiciones de vida de la población [3]. Este trabajo de investigación se realizará con el propósito de realizar el monitoreo y seguimiento de los convenios de Restauración Forestal con Fines de Conservación Ambiental y Protección de Cuencas Hídricas suscritos entre el Ministerio del Ambiente y los Gobiernos Autónomos Descentralizados Parroquiales de los cantones Colta, Guamote, Alausí y Chunchi, esto debido a la necesidad de verificar el cumplimiento de las disposiciones legales, reglamentarias y normativas establecidas en los convenios

\section{Materiales y Métodos}

La presente investigación se desarrolló en las áreas del Programa Nacional de Reforestación propuestas por los GADs parroquiales Santiago de Quito convenio Nro. MAESPN-RF-GAD-0277, Palmira convenio Nro. MAE-SPN-RF-GAD-0037, Pistishí convenio Nro. MAE-SPN-RF-GAD-0136 y Compud convenio Nro. MAE-SPN-RF-GAD-0101.

\subsection{Metodología}

\subsubsection{Inspección de superficie plantada}

El proceso de verificación de las plantaciones se realizó con la metodología emitida desde central, en la que se determinó entre las prácticas de plantación en bloque y plantación en línea, de cada modalidad se seleccionó una muestra aleatoria equivalente al $10 \%$ del total de la superficie y el $10 \%$ al total de predios. La variable de medición 
dentro del muestreo se definió como una variable cualitativa de presencia o ausencia de plantación en cada uno de los predios. Para ubicar los puntos de observación dependió de la práctica aplicada; para fines de verificación cualitativa, las prácticas se clasificaron en dos categorías: Plantación en bloque (PB) y plantación en línea (PL).

\subsubsection{Medición en plantación en bloque (PB)}

Se aplicó para aquellas prácticas que coinciden con la plantación en bloque, para cada desde central fue establecido 10 puntos fijos que fueron ubicados en campo con un GPS, en este tipo de verificación cualitativa se registró si hubo o no evidencia de plantación.

\subsubsection{Mediciones en Plantación en Línea (PL)}

Se aplicó para las prácticas que coinciden con la plantación de árboles en líneas, este recorrido fue segmentado en diez (10) puntos fijos los cuales fueron ubicados en campo con GPS. Desde cada uno de los puntos se realizó una observación en dirección de la plantación con un radio de visión aproximado de 20 m para verificar de forma cualitativa la plantación.

\subsubsection{Verificación de las áreas para la restauración}

Para la verificación de los predios pala la restauración, los puntos fueron emitidos desde Central los cuales fueron ubicados en campo con GPS; en cada uno de los puntos de observación se tomó fotografías como evidencias. La inspección de campo consistió en la valoración de cada uno de los predios, en base a recorridos realizados se consideró si las áreas verificadas son o no aptas para el proceso de restauración.

Para las inspecciones en campo se estableció brigadas conformadas por técnicos del Programa Nacional de Restauración asignado a la provincia de Chimborazo, un representante del GAD parroquial y un estudiante de la Carrera de Ingeniería Forestal de la ESPOCH, para el registro de la información se utilizó como herramienta de trabajo las fichas de campo generadas con la metodología y protocolos de evaluación; durante los recorridos, con un GPS de navegación se creó un Track. En cada predio inspeccionado se tomó fotografías una por cada dirección de los puntos cardinales (Norte, Sur, Este y Oeste) con la finalidad de tener una visión amplia que permita identificar el estado actual de las áreas verificadas; la codificación de cada fotografía se la realizó en base al Manual para la verificación cualitativa emergente del establecimiento del Programa Nacional de Reforestación. 


\section{Resultados y Discusión}

\subsection{Parroquial palmira}

Las brigadas formadas recorrieron 51,23 ha de un total de 499,79 ha, comprometidas en el convenio MAE-SPN-RF-GAD-0037 (Figura 1). La superficie muestreada fue distribuida en 57 predios de un total de 721 entre las modalidades de plantación en bloque y plantación en línea (Tabla 1).

\subsubsection{Superficie plantada}

\section{Medición en plantación en bloque (PB)}

En esta modalidad se evaluó una total de 7,91 ha, toda esta superficie presenta actividades de plantado; con respecto al número de predios muestreados en bloque son 3 , de la misma forma todos los predios presentan acciones de plantado (Tabla 2).

\section{Mediciones en plantación en línea (PL)}

Con respecto superficie plantada en línea se ubicaron 43,32 ha de las cuales 30,48 ha se observó indicios de haberse realizado la plantación (Tabla 3), lo que corresponde al $59,50 \%$; mientras tanto la superficie no plantada fue 122,84 ha lo que equivale al $25,06 \%$.

\subsubsection{Resultados generales}

De la muestra seleccionada, 38,39 ha (74,94\%) se evidenció actividades de plantación y 12,84 ha $(26,06 \%)$ no se observó plantación alguna. Se registro un total de 57 predios, de las cuales 41 presentó acciones de plantado, esto representa el 71,93\% de la muestra. En 16 predios no se identificó indicios actividades de plantación lo cual representa el $28,07 \%$ de la muestra total (Tabla 4).

No todos los predios evaluados registraron el $100 \%$ de su superficie plantada. De los 10 puntos de control establecidos en cada predio, 16 predios se encuentran plantado en su totalidad, 16 predios no poseen indicios de plantación en ninguno de los puntos de control. La mayor superficie plantada corresponde al predio No. 000JBR con área de 7,50 ha; seguido del predio No. 000168 con un área de 1,94 ha (Figura 2).

\subsection{Parroquia pistishi}

En la verificación de las plantaciones se recorrió 38,66 ha de un total de 200,49 ha (Figura 3), la superficie muestreada fue distribuida en 29 predios de un total de 314 entre las modalidades de plantación en bloque y plantación en línea (Tabla 5). 


\subsubsection{Superficie plantada}

\section{Medición en plantación en bloque (PB)}

En la Tabla 6 se observa que la superficie total en bloque es de 27,94 ha, toda esta superficie presenta actividades de plantación. Con respecto al número de predios muestreados en bloque es 1, el cual presenta indicios de plantación (Tabla 6).

\section{Mediciones en plantación en línea (PL)}

Con respecto superficie plantada en línea se ubicaron 10,72 ha de las cuales 8,33 ha se encontraron plantadas (Tabla 7), lo que corresponde al 21,54\%, mientras que la superficie no plantada fue 2,39 ha lo que equivale al $6,19 \%$.

\subsubsection{Evaluación general}

De la muestra seleccionada, 36,27 ha $(93,81 \%)$ se evidenció actividades de plantación y 2,39 ha $(6,19 \%)$ no se observó plantación alguna. Se registro un total de 29 , de las cuales 22 presentó acciones de plantado, esto representa el 75,86\% de la muestra. En 7 predios no se identificó indicios de plantado, lo cual representa el 24,14\% de la muestra total (Tabla 8).

No todos los predios identificados registraron el $100 \%$ de su superficie plantada. De los 10 puntos de control establecidos en cada predio, 6 predios se evidenciaron plantado en su totalidad, 16 predios no se encontraron plantado en todos sus puntos y 7 predios no presentaron indicios de plantación. La mayor superficie plantada corresponde al predio No. 000MOF con área de 0,60 ha; y predio No. 000M0G con un área de 0,43 ha (Figura 4).

\subsection{Parroquia Santiago de quito}

\subsubsection{Superficie evaluada}

Se verificó 67 predios que abarca un área de 67,78 has. Las áreas evaluadas se las considero aptas para la reforestación, esto debido a que los suelos se encuentran en proceso de degradación; durante el recorrido, también se observó en los predios presencia de labores agrícolas, predios en centros poblados y con presencia de vegetación arbustiva y Arborea. En la siguiente tabla se observa resultados del recorrido por varios predios propuesto por el GAD Santiago de Quito (Figura 5). 
Table 1

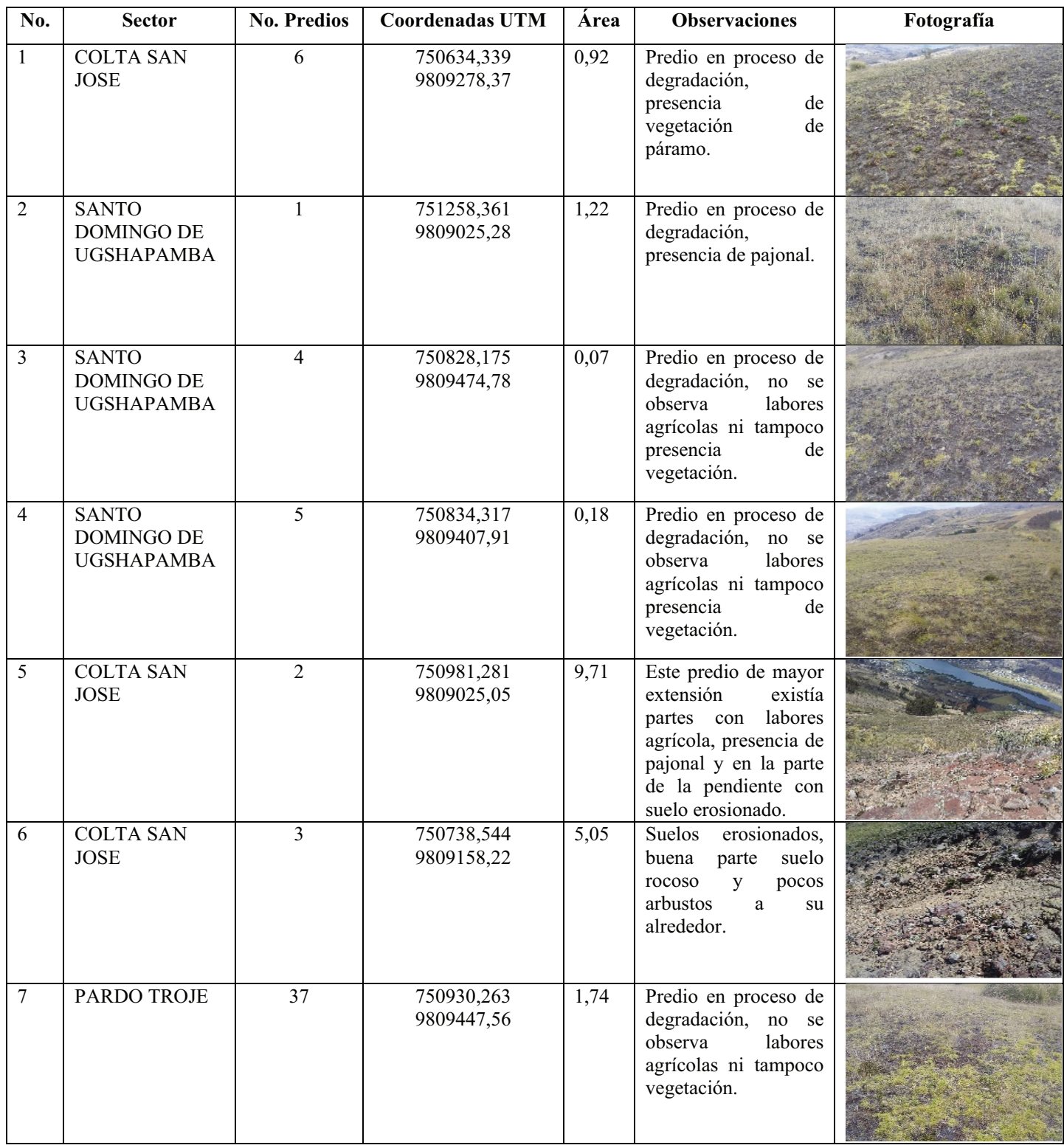

\subsection{Parroquia Compud}

\subsubsection{Superficie evaluada}

Se evaluó 13 predios que abarca un área de 7,27 has; los predios evaluados fueron considerado aptos para la reforestación, el objetivo del GAD fue reforestar para uso de sombra para ganado. En la siguiente tabla se observa los resultados de la evaluación de los predios en el GAD Compud (Figura 6). 
Table 2

\begin{tabular}{|c|c|c|c|c|c|c|}
\hline No. & Sector & Coordenadas UTM & Área & Predio & Observaciones & Fotografías \\
\hline 1 & SAGUIN & $\begin{array}{l}732172 \\
9739559\end{array}$ & 0,14 & 000M7X & $\begin{array}{ll}\text { Presencia } & \text { de } \\
\text { vegetación } & \\
\text { arbustiva. } & \end{array}$ & \\
\hline 2 & SAGUIN & $\begin{array}{l}732275 \\
9739515\end{array}$ & 0,37 & $000 \mathrm{M} 86$ & $\begin{array}{l}\text { Presencia de } \\
\text { de pultivo } \\
\text { ganado. }\end{array}$ & \\
\hline 3 & CULLUN & $\begin{array}{l}731144 \\
9737652\end{array}$ & 0,27 & 000M8A & $\begin{array}{l}\text { Predio de } \\
\text { acceso. }\end{array}$ & \\
\hline 4 & SAGUIN & $\begin{array}{l}732450 \\
9739768\end{array}$ & 1,13 & $000 \mathrm{M} 8 \mathrm{~J}$ & $\begin{array}{l}\text { Presencia de cultivo } \\
\text { de pasto en predio } \\
\text { inspeccionado. }\end{array}$ & \\
\hline 5 & SAGUIN & $\begin{array}{l}732123 \\
9739876\end{array}$ & 0,16 & $00 \mathrm{M} 8 \mathrm{M}$ & $\begin{array}{lr}\text { Predio } & \text { con } \\
\text { presencia de } & \text { árboles } \\
\text { plantados } & \text { y } \\
\text { presencia de } & \text { cultivo } \\
\text { de pasto prar para } \\
\text { ganado. }\end{array}$ & \\
\hline 6 & SAGUIN & $\begin{array}{l}732123 \\
9739876\end{array}$ & 0,15 & $000 \mathrm{M} 8 \mathrm{~N}$ & $\begin{array}{l}\text { Predio con presencia } \\
\text { de cultivo de pasto } \\
\text { para ganado. }\end{array}$ & \\
\hline 7 & SAGUIN & $\begin{array}{l}731857 \\
9739825\end{array}$ & 1,54 & $000 \mathrm{M} 8 \mathrm{O}$ & $\begin{array}{l}\text { Predio con presencia } \\
\text { de árboles plantados } \\
\text { en línea. }\end{array}$ & \\
\hline
\end{tabular}




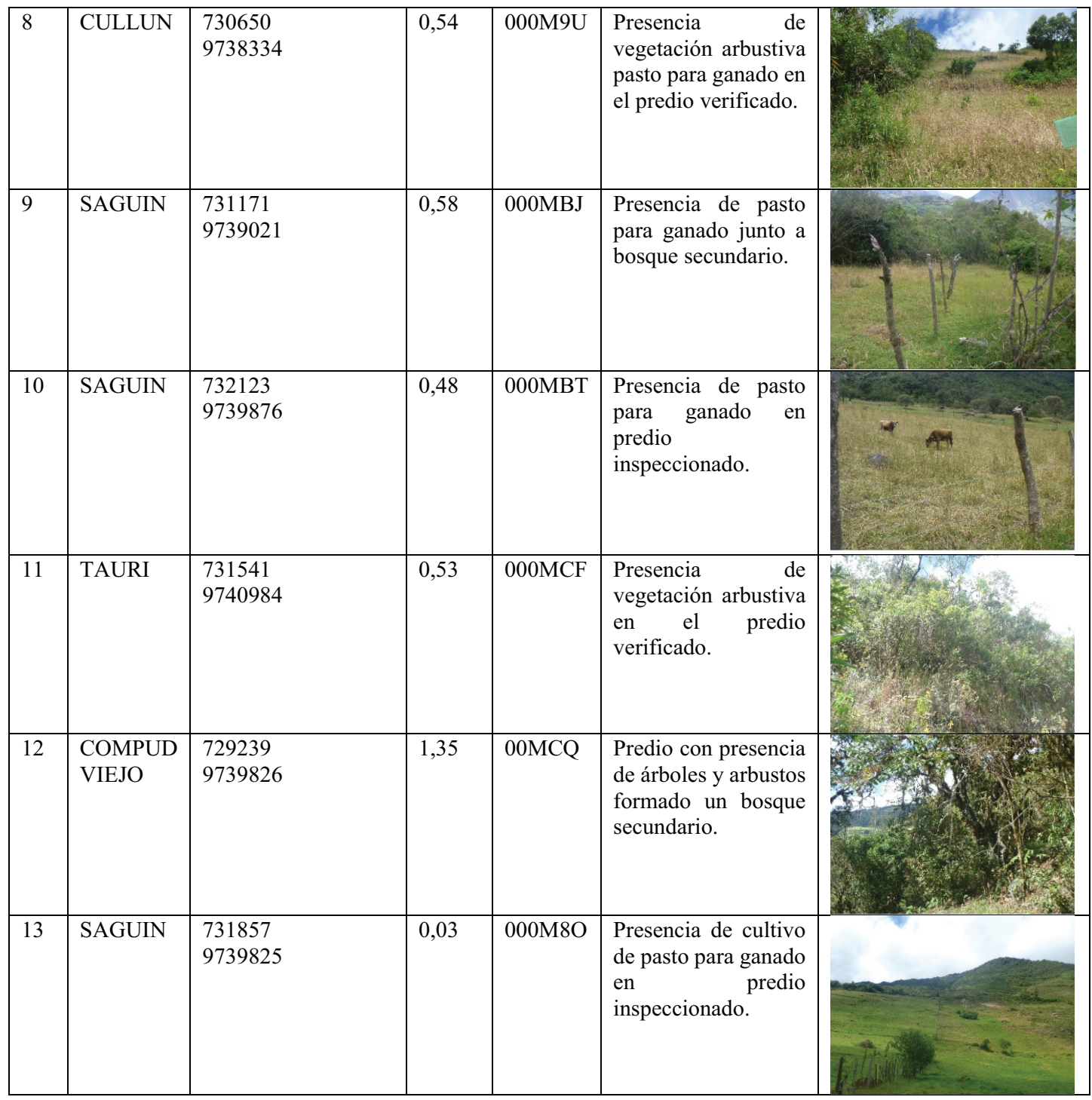

Table 3

Superficie y número de predios verificados en la parroquia Palmira.

\begin{tabular}{|l|l|l|l|l|l|l|} 
Modalidad & $\begin{array}{l}\text { Superficie } \\
\text { total }\end{array}$ & $\begin{array}{l}\text { Área } \\
\text { muestreada }\end{array}$ & \% Muestra & $\begin{array}{l}\text { Predios } \\
\text { totales }\end{array}$ & $\begin{array}{l}\text { Predios } \\
\text { muestra }\end{array}$ & $\begin{array}{l}\% \\
\text { Muestra }\end{array}$ \\
\hline PB & 7,91 & 7,91 & 15,44 & 3 & 3 & 5,26 \\
\hline PL & 491,88 & 43,32 & 84,56 & 718 & 54 & 94,74 \\
\hline Total & 499,79 & 51,23 & 100 & 721 & 57 & 100
\end{tabular}

\section{Conclusiones}

La deforestación en el Ecuador es una problemática que demanda acciones concretas y sostenibles por parte de las autoridades competentes al revisar datos históricos se encuentran que en el periodo 1990-2000 la deforestación promedio fue de 89,944 


\section{Table 4}

Superficie plantada y no plantada mediante la modalidad en bloque, parroquia Palmira.

\begin{tabular}{l|l|l|l|l|}
$\begin{array}{l}\text { Tipo de } \\
\text { plantación }\end{array}$ & Superficie $\mathbf{( H a )}$ & Superficie (\%) & Número de predios & Predios (\%) \\
\hline $\begin{array}{l}\text { Plantado } \\
\text { No plantado }\end{array}$ & $\mathbf{7 , 9 1}$ & 15,44 & 3 & 5,26 \\
\hline Total & 0 & 0 & 0 & 0 \\
\hline
\end{tabular}

Table 5

Superficie plantada y no plantada mediante la modalidad en línea, parroquia Palmira.

\section{Tipo de plantación}

Plantado

No plantado

Total

Table 6

Superficie $(\mathbf{H a})$
30,48
12,84
43,32

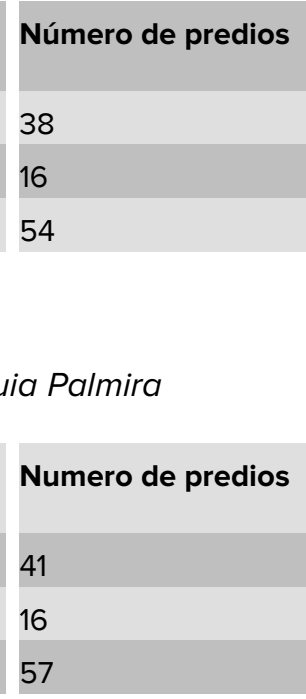

Predios (\%)

66,67

28,07

94,74

Numero de predios plantados y no plantados parroquia Palmira

\begin{tabular}{l|l|l|l|l|}
$\begin{array}{l}\text { Tipo de } \\
\text { plantación }\end{array}$ & Superficie $(\mathbf{H a )}$ & Superficie (\%) & Numero de predios & Predios (\%) \\
\hline Plantado & 38,39 & 74,94 & 41 & 71,93 \\
\hline No plantado & 12,84 & 25,06 & 16 & 28,07 \\
\hline Total & 51,23 & 100,00 & 57 & 100,00 \\
\hline
\end{tabular}

\section{Table 7}

Superficie y numero de predios verificados en la parroquia Pistishi.

\begin{tabular}{|l|l|}
\multicolumn{2}{|c|}{$\begin{array}{c}\text { ModalidacSuperficie } \\
\text { total }\end{array}$} \\
\hline PB & 99,78 \\
\hline PL & 100,7 \\
\hline Total & 200,49 \\
\hline
\end{tabular}

$\begin{aligned} & \text { Área } \\ & \text { muestreada }\end{aligned}$
27,94
10,72
38,66

\% Muestra
72,27
27,73
100

$\begin{aligned} & \text { Predios } \\ & \text { totales }\end{aligned}$
5
309
314

Predios
muestra
1
28
29

\% Muestra

3,45

96,55

100

Table 8

Superficie plantada y no plantada mediante la modalidad en bloque, parroquia Pistishi.

Tipo de
plantación
Plantado
No plantado
Total

Superficie $(\mathbf{H a})$
27,94
0
27,94

Superficie (\%)
72,27
0
72.27

\begin{tabular}{|l|l|} 
Número de predios & Predios (\%) \\
\hline 1 & 3,45 \\
\hline 0 & 0 \\
\hline 1 & 3,45 \\
\hline
\end{tabular}

ha/año para una tasa de deforestación de -0,71\%, mientras que para el período 2000 2008 la deforestación promedio fue de 77,647 ha/año para una tasa de $-0,66 \%$. Por 


\section{Table 9}

Superficie plantada y no plantada mediante la modalidad en línea, parroquia Pistishi.

\begin{tabular}{l|l|l|l|l|} 
Tipo de plantación & Superficie $(\mathrm{Ha})$ & Superficie (\%) & $\begin{array}{l}\text { Número de } \\
\text { predios }\end{array}$ & Predios (\%) \\
\hline Plantado & 8,33 & 21,54 & 21 & 72,41 \\
\hline No plantado & 2,39 & 6,19 & 7 & 24,14 \\
\hline Total & 10,72 & 27,73 & 28 & 96,55 \\
\hline
\end{tabular}

Table 10

Número de predios plantados y no plantados parroquia Pistishi.

Tipo de
plantación
Plantado
No plantado
Total

Superficie $(\mathbf{H a})$
36,27
2,39
38,66

Superficie (\%)
93,81
6,19
100,00

Número de predios
22
7
29

Predios (\%)

75,86

24,14

100,00

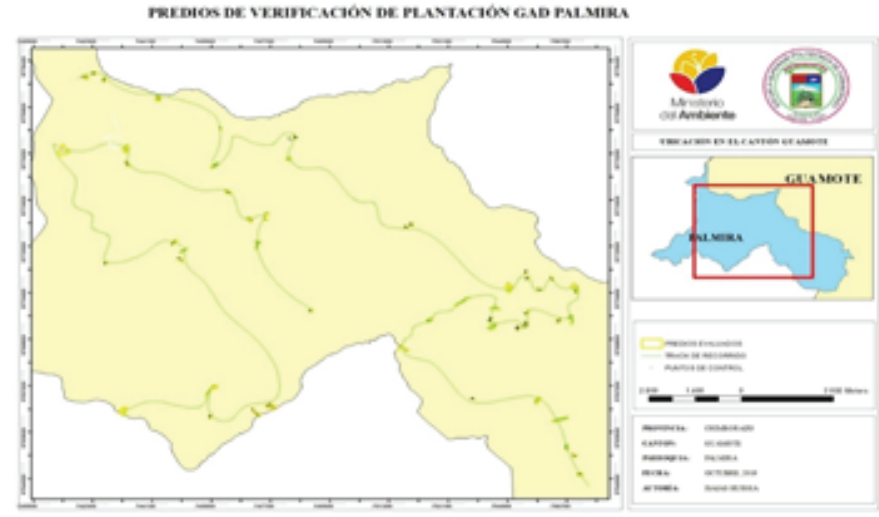

Figure 1

Ubicación de los predios muestreados en la parroquia Palmira.

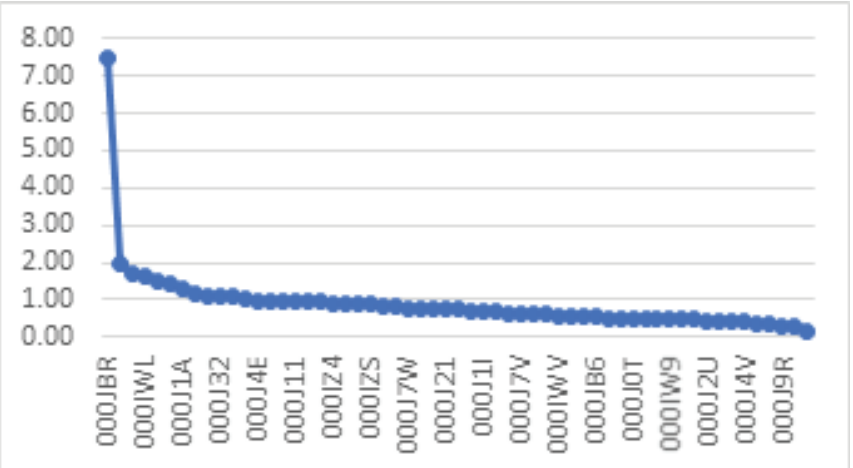

\section{Figure 2}

Porcentaje de superficie verificada por predio.

otro lado, durante el período 2008-2012 la deforestación fue de 65,880 ha/año para 


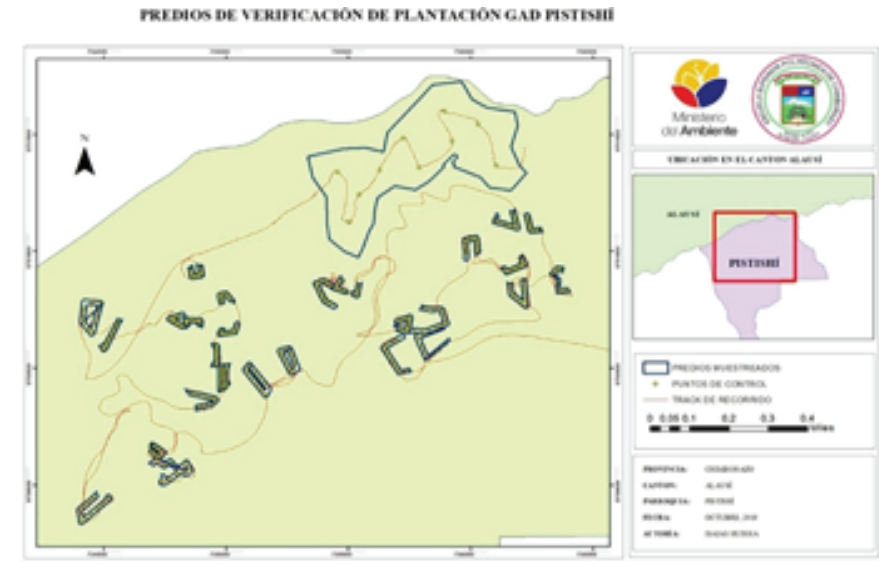

Figure 3

Ubicación de los predios muestreados en la parroquia Pistishi.

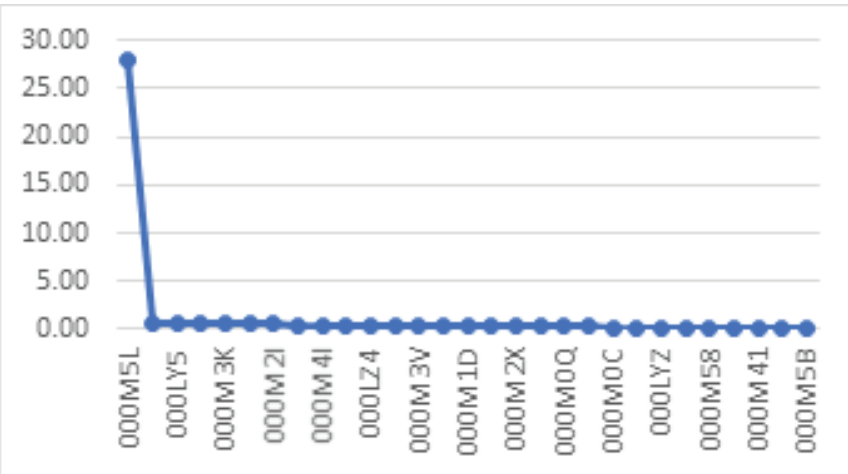

Figure 4

Porcentaje de superficie verificada por predio.

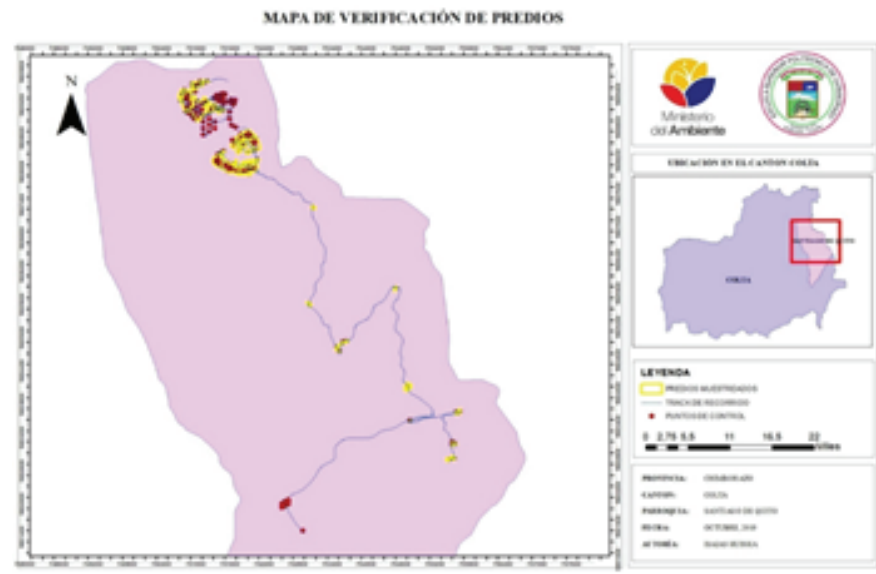

Figure 5

Áreas propuestas por la parroquia Santiago de Quito.

una tasa de -0,54\%, datos tomados del Proyecto mapa histórico de deforestación MAE 2012, siendo esta investigación el inicio para el programa de restauración forestal con 


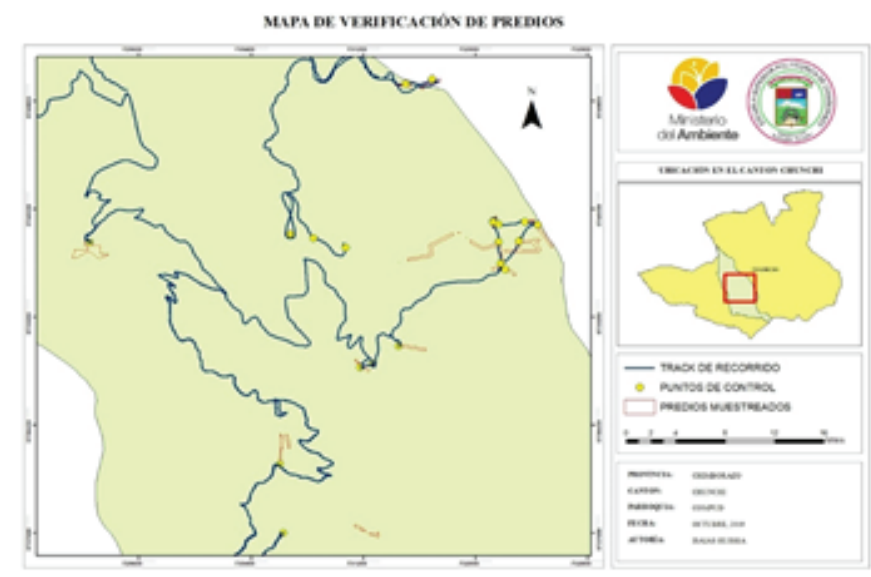

Figure 6

Áreas propuestas por la parroquia Compud.

el fin recuperar la cobertura vegetal, a través de un manejo integral del paisaje forestal, para la los recursos naturales y mejorar las condiciones de vida de la población, con estos antecedentes podemos concluir que en el GAD parroquial Palmira se estimó al área plantada de $74,94 \%$ equivalente a 39,38 ha en el que se registró indicios de plantado y el $26,06 \%$ equivalente a 12,84 ha no presento indicios de plantado; con relación al número de predios verificados se observó que de 57 predios 14 equivalente a $71,93 \%$ presentó acciones de plantado mientras que 16 predios equivalentes a $28,07 \%$ no se registra indicios de plantado. En el GADPR Pistishi se estima que la superficie plantada es del 93,81\% equivalente al 36,27 ha se registró indicios de plantado y el 6,19\% equivalente a 2,39 ha no presento indicios de plantado de la muestra verificada; con relación al número de predios verificados se observó que de 29 predios 22 equivalente a $75,86 \%$ presentó acciones de plantado mientras que 2 predios equivalentes a $24,14 \%$ no se registra indicios de plantado. En el GAD parroquial Compud los predios evaluados se consideraron aptos para la reforestación, esto debido a que el objetivo del GAD es realizar plantaciones para otorgar sombra para el ganado.

El objetivo del Ministerio del Ambiente (MAE) es que la tasa de deforestación sea cero, esto quiere decir que la deforestación sea equivalente a la Restauración Forestal, el MAE entre uno de sus objetivos era reforestar al 2017 al menos 220,000 ha para el período 2014-2017 de acuerdo con las estimaciones de la deforestación acumulada esperada en aquel entonces. En la realidad y como se puede apreciar en la investigación realizada los Gobiernos Autónomos que firmaron los convenios no lo pudieron cumplir con el $100 \%$ de la restauración forestal, por la no entrega de presupuesto por parte del MAE hacia los GADS, estos desembolsos se realizaron por etapa cumplida, es así que para el manejo de las plantaciones no existió presupuesto, es por eso que el resultado no es de un $100 \%$, sabiendo que los GADS parroquiales al disponer de pocos recursos no pudieron sostener este proyecto. Pero hay que considerar que las actividades realizadas son un aporte a la recuperación de cobertura forestal que ha sido deforestada históricamente, este es un buen escenario para continuar con el proceso, establecer nuevas alianzas estratégicas a nivel de gobierno y ONG's que 
permitan continuar en los métodos de conservación que gestiona el MAE, los Gobiernos Provinciales y Parroquiales para la biodiversidad, la protección de agua y suelos, disminución de riesgos por deslizamientos e inundaciones al tener mejor cobertura boscosa.

\section{References}

[1] Vallauri D, Aronson J, Barbéro M. An analysis of forest restoration 120 years after reforestation of badlands in the South-western Alps [Internet]. Restoration Ecology. 2002. 10:16-26. Disponible en: https://onlinelibrary.wiley.com/doi/abs/10.1046/j.1526-100X.2002.10102.x

[2] Acuerdo Ministerial. Normas para el manejo sustentable de los bosques andinos [Standards for the sustainable management of Andean forests, Internet]. No 128; 2006. Disponible en: http://ecuadorforestal.org/wp-content/uploads/2010/05/ NORMAS-PARA-ELMANEJO-SUSTENTABLE-DE-LOSBOSQUES-ANDINOS.pdf. Spanish

[3] MAE. Ministra Lorena Tapia suscribió convenios de restauración forestal con 46 GAD [Minister Lorena Tapia signed forest restoration agreements with 46 GAD,Internet]. 2014. Disponible en: http://www. ambiente.gob.ec/ministra-Iorena-tapia-suscribio-convenios-de-restauracion-forestal-con-46-gads/. Spanish

[4] Acuerdo Ministerial. Plan Nacional de Restauración Forestal 2014-2017 [National plan of forest restoration,Internet]. No 041; 2014. Disponible en: http://sociobosque.ambiente.gob.ec/files/images/ articulos/archivos/amr041.pdf. Spanish

[5] Acuerdo Ministerial. Manual operativo para la implementación del incentivo económico para la restauración forestal [Operational manual for the implementation of the economic incentive for forest restoration,Internet]. No 211; 2014. Disponible en: https://www.derechoecuador.com/registro-oficial/ 2014/08/registro-oficial-no-317---viernes-22-de-agosto-de-2014-segundosuplemento. Spanish

[6] Acuerdo Ministerial No. 315. Expídanse las reformas al Acuerdo Ministerial Nro. 211 [Issue the reforms to the ministerial agreement,Internet]. 2014 jul 21. Obtenido de: https://vlex.ec/vid/ expidense-reformas-acuerdo-ministerial-551529474. Spanish

[7] Acuerdo Ministerial No. 065. Sustituir el manual operativo para la implementación del incentivo económico para la restauración forestal [Replacing the operative manual for the implementation of the economic incentive for forest restoration,Internet]. 2015. Obtenido de: http://sociobosque.ambiente. gob.ec/files/images/articulos/archivos/AM065R.pdf. Spanish

[8] FAO. Conjunto de herramientas para la gestión forestal sostenible (GFS) [Toolkit for sustainable forest management,Internet]. 2014. Disponible en: https://bit.ly/2gcwUMc. Spanish

[9] FAO. Evaluación de los recursos forestales mundiales [Global forest resources assessment,Internet].Ecuador: Informe Nacional; 2015. Obtenido de: http://www.fao.org/3/a-az203s.pdf. Spanish

[10] FAO. Evaluación de los recursos forestales mundiales 2000 [Evaluación de los recursos forestales mundiales 2000,Internet]. Informe Principal; 2002. Disponible en: http://www.fao.org/3/y1997s/ y1997s00.htm. Spanish

[11] Freitas SR, Neves CL, Chernicharo P. Tijuca National Park: two pioneering restorationist initiatives in Atlantic forest in southeastern Brazil. Brazilian Journal of Biology. 2006;66:975-982. Disponible en: http://www.scielo.br/pdf/bjb/v66n4/03.pdf

[12] Kimmins JP. Ecosystem management and landscape ecology: The ultimate focus in forest ecology. $3^{\text {rd }}$ ed. In Forest ecology. A foundation for sustainable forest management and environmental ethics in forestry, Chap 19. 2003. Upper Saddle River, N.J., Prentice Hall. En prensa.

[13] MAE. Restauración y conservación forestal, una nueva visión de desarrollo [Forest restoration and conservation, a new vision of development,Internet]. 2015. Disponible en: http://www.ambiente.gob. ec/restauracion-y-conservacion-forestal-una-nueva-vision-de-desarrollo/. Spanish

[14] MAE. Bosques protectores. Sistema nacional de áreas protegidas del Ecuador [National system of protected areas of Ecuador,Internet]. 2015. Disponible en: http://areasprotegidas.ambiente.gob.ec/es/ content/bosques-protectores. Spanish 